\title{
Effects of Lactobacillus cultures on performance and egg quality during the early laying period of hens
}

\author{
R. Kalavathy ${ }^{1}$, W. Abdullah ${ }^{2}$, S. Jalaludin ${ }^{3}$, C.M.V.L. Wong ${ }^{4}$ \\ and Y.W. Ho ${ }^{1,5}$ \\ University Putra Malaysia, \\ ${ }^{I}$ Institute of Bioscience, \\ ${ }^{2}$ Department of Biochemistry, Microbiology, \\ ${ }^{3}$ Department of Animal Science \\ 43400 UPM, Serdang, Selangor, Malaysia \\ ${ }^{4}$ University of Malaysia Sabah \\ 88999 Kota Kinabalu, Sabah Malaysia
}

(Received 9 June 2004; revised version 16 March 2005; accepted 4 August 2005)

\begin{abstract}
A feeding experiment was conducted to evaluate the effects of a mixture of 12 Lactobacillus strains (LC) on the performance and egg quality of laying hens for 16 weeks, from 20 to 35 weeks of age. One hundred and forty four 16-week-old Lohmann Brown pullets were assigned randomly to two dietary treatments: 1 . a basal diet (control) and 2. a basal diet $+1 \mathrm{~g} \mathrm{~kg}^{-1}$ LC. Each dietary treatment had four replicates with 18 cages per replicate (one hen per cage).

The feed efficiency was significantly $(\mathrm{P}<0.05)$ improved in LC-fed hens when compared to control hens throughout the experimental period despite the fact that there was no significant difference in feed intake between the treatments.

LC-fed hens had significantly $(\mathrm{P}<0.05)$ higher egg production and greater egg mass than control hens from 28 to 35 weeks of age, but no significant differences were observed between treatments from 20 to 27 weeks of age. The proportions of small and peewee eggs of hens receiving the LC diet were significantly smaller as compared to those of the control hens (22 vs $28 \%$ ) from 20 to 35 weeks of age. However, the internal egg quality (Haugh unit), shell quality (egg specific gravity and shell thickness) and the yolk colour were not improved by the supplementation of LC to hens, from 20 to 35 weeks of age.

The results from the present study demonstrated that LC could effectively improve feed efficiency, egg weight and egg size during the early stage of egg production cycle from 20 to 35 weeks of age.
\end{abstract}

KEY WORDS: probiotics, Lactobacillus, laying hens, performance, egg quality

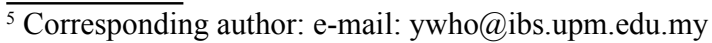

